\begin{tabular}{|l|l|}
\hline Title: & Control Strategy for Modular Multilevel Matrix Converters at High Output Frequencies \\
\hline Authors: & Braeckle, Dennis; Himmelmann, Patrick; Schnarrenberger, Mathias; Hiller, Marc \\
\hline Institute: & $\begin{array}{l}\text { Karlsruhe Institute of Technology (KIT) } \\
\text { Elektrotechnisches Institut (ETI) } \\
\text { Power Electronic Systems }\end{array}$ \\
\hline Type: & Conference Proceedings \\
\hline Published at: & Energy Conversion Congress and Exposition (ECCE), 2017 IEEE \\
\hline Hyperlinks: & DOI: 10.1109/ECCE.2017.8096422 \\
\hline
\end{tabular}

(c) 2017 IEEE. Personal use of this material is permitted. Permission from IEEE must be obtained for all other uses, in any current or future media, including reprinting/republishing this material for advertising or promotional purposes, creating new collective works, for resale or redistribution to servers or lists, or reuse of any copyrighted component of this work in other works. 


\title{
Control Strategy for Modular Multilevel Matrix Converters at High Output Frequencies
}

\author{
Dennis Braeckle*, Patrick Himmelmann*, Mathias Schnarrenberger* and Marc Hiller* \\ *Institute of Electrical Engineering (ETI) \\ Karlsruhe Institute of Technology, Karlsruhe, Germany \\ Email: dennis.braeckle@kit.edu
}

\begin{abstract}
This paper presents a new control strategy for Modular Multilevel Matrix converters where the output frequency is higher then the input frequency. In operating points when $\omega_{\text {out }} \geq \omega_{\text {in }}$ the energy pulsation in the converter cells are dominated by the second harmonic of the input frequency. These energy pulsations can be identified and compensated by inducing additional currents. The results are verified by simulation for a laboratory scaled prototype of the Modular Multilevel Matrix Converter.
\end{abstract}

\section{INTRODUCTION}

The Modular Multilevel Matrix Converter (M3C) is a direct $\mathrm{AC}$ to $\mathrm{AC}$ converter based on the principle of the Modular Multilevel Converter (MMC). The M3C is in the focus of recent research activities as a converter for low speed, high torque applications ([1]-[4]). The $\mathrm{M} 3 \mathrm{C}$ is also capable of driving a load with equal input and output frequencies if the output power is reduced ([4]-[6]). Figure 1 depicts the basic structure of the M3C system. The M3C connects two 3-phase AC systems with 9 arms $\left(v_{11} . . v_{33}\right)$ and coupling inductors $L$. Each arm is composed of a series connection of $N$ full bridge cells, each including a cell capacitor $C$. They act as voltage sources as depicted in fig. 1 .

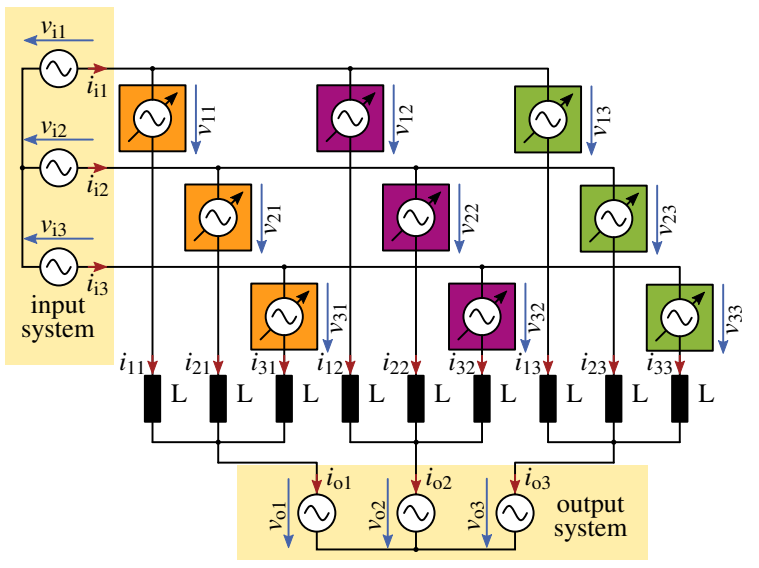

Fig. 1. Schematic overview of the Modular Multilevel Matrix Converter

\section{A. Basic Control Strategies for the M3C}

The control strageties of the M3C system have been subject to recent research activities. [7] shows that the M3C has advantages regarding the energy pulsation compared to a conventional MMC back-to-back configuration if the output frequeny is greater than the input frequency. In [5], [6] the control strategies for the whole output frequency range of the $\mathrm{M} 3 \mathrm{C}$ are presented. When the input and output frequencies are similar, additional control effort is necessary to balance the arm energies. As shown in [4], [8] stable operation in these points is possible with reduced output power capability. As shown in [3], [5], [6] the energy pulsation of each converter arm can be reduced when the output frequency is smaller than the input frequency.

However, at high output frequencies the major energy pulsation occurs with twice the input frequency [8]. The approach presented in this paper illustrates a possible way to compensate the energy pulsation caused by the input side for positive and negative output frequencies. First the occurring pulsation is determined analytically. Second a control approach is presented to reduce this pulsation. The currents that are necessary for this control method cause additional losses in the semiconductors, however the cell capacitors can be designed smaller. An optimization of the factor to reduce the pulsation depending on losses and cell volume is shown. Simulations for a laboratory scaled prototype are presented to verify this control approach.

\section{Fundamentals}

Figure 1 shows the overall system of the M3C. The aim is to control the output power, the input power and the energy distribution among all converter arms. In consequence the 9 arm currents $i_{x y},[x, y \in\{1,2,3\}]$ and 9 arm energies $w_{x y},[x, y \in\{1,2,3\}]$ have to be controlled. The arm voltages $v_{x y},[x, y \in\{1,2,3\}]$ are used to induce the arm currents. Each arm current can be separated into a part of the input current $\frac{i_{\text {in }}}{3}$, a part of the output current $\frac{i_{\text {out }}}{3}$ and internal currents that are effective neither to the input side nor the output side. The arm voltages in combination with the arm currents produce the arm power $p_{x y},[x, y \in\{1,2,3\}]$. In order to keep the cell capacitor energies constant, the mean power has to be 0 . To determine a suitable control scheme, the system can be transformed and decoupled to control each arm energy component separately [5].

\section{A. Transformation of Parameters}

The arm currents, voltages, energies and powers are transformed to achieve a decoupled system and to find degrees of freedom to reduce the energy pulsations. The transformation 
is a sequence of Clarke transforms and a sum/difference operation as shown in [5]. The 9 currents, voltages and powers are transformed in eq. (1) (see section VI for transformation matrices).

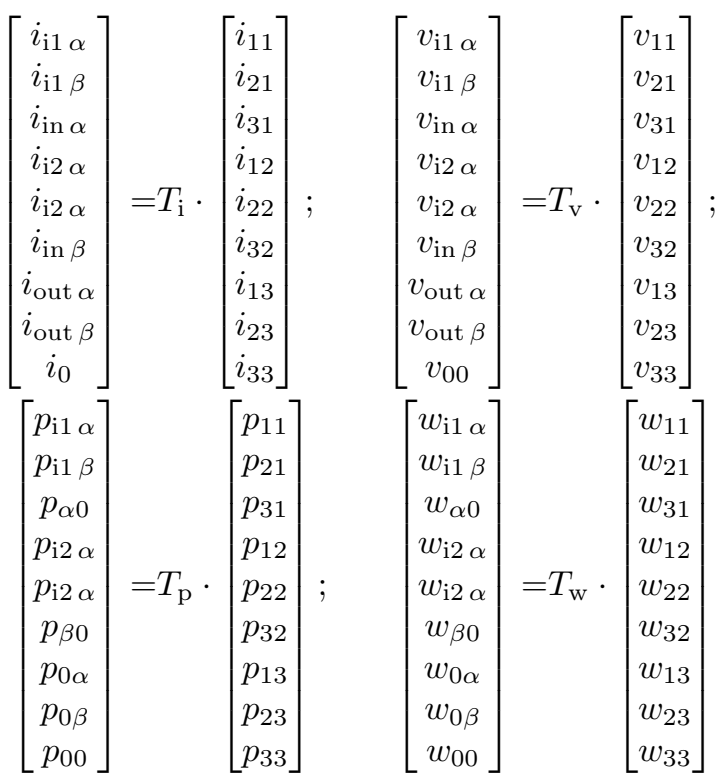

As a result of the transformation the components $x y$ are decoupled. The input currents $i_{\text {in } \alpha \beta}$ and output currents $i_{\text {out } \alpha \beta}$ are separated from the two internal current systems $i_{\mathrm{i} 1 \alpha \beta}$ and $i_{\mathrm{i} 2 \alpha \beta}$. Hence, they can be controlled independently. The corresponding voltages $v_{x y}$ and powers $p_{x y}$ are transformed in the same manner. The arm energies $w_{x y}$ can be calculated with the measured capacitor voltages $v_{C, x y}$ by

$$
w_{x y}=\frac{1}{2} C_{\text {arm }} v_{C, x y}^{2}
$$

\section{B. Transformed Circuit Equivalent Diagram}

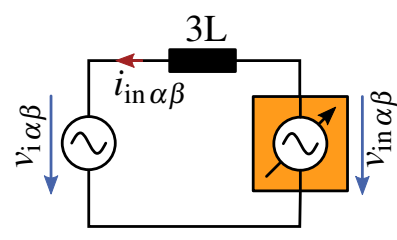

(a)

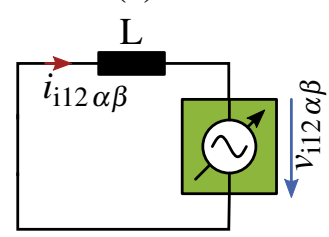

(c)

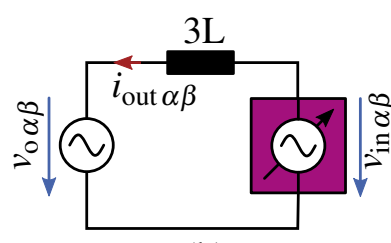

(b)

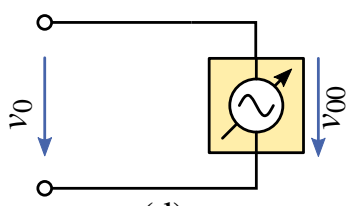

(d)
Fig. 2. Transformed decoupled circuit equivalent diagram: (a) input side (b) output side (c) internal circuits (d) zero sequence system

When applying eq. (1) to the measured values of the converter, the complex coupled structure of fig. 1 can be broken into 4 decoupled equivalent circuits (see fig. 2). Figure 2 (a) and Figure 2 (b) show the resulting circuits for the input side and the output side respectively. The transformed arm voltages $v_{\text {in } \alpha \beta}$ can be used to control the current $i_{\text {in } \alpha \beta}$ and therefore the power $p_{\text {in } \alpha \beta}$ exchanged with the input side. The output power $p_{\text {out } \alpha \beta}$ of the converter can be controlled independently with the transformed arm voltages $v_{\text {out } \alpha \beta}$ and currents $i_{\text {out } \alpha \beta}$. In addition there are four transformed arm voltages $v_{\mathrm{i} 12 \alpha \beta}$ and currents $i_{\mathrm{i} 12 \alpha \beta}$ that are neither effective on the input nor the output side. The equivalent diagram is shown in fig. 2 (c). The corresponding arm powers $p_{\mathrm{i} 12 \alpha \beta}$ do not exchange any power with the terminals of the system. Furthermore the internal power components can be used to exchange energy among the converter arms to ensure an equal energy distribution among the arms. A zero sequence voltage $v_{00}$ can be applied as an additional degree of freedom if the neutral points of input and output system are not connected. The zero sequence voltage is needed in operationg points with similar input and output frequencies ([1], [2], [4]). The decoupled system allows a simple current controller design for each of the components [8].

\section{Energy Control of the System}

The energy control of the system is divided up into two parts. Within one arm the internal modulation scheme ensures that each cell of one arm is stressed equally. The 9 overlaying energy controllers only control the overall arm energies $w_{x y}$. According to eq. (1) and fig. 2 the arm energies $w_{x y}$ can be influenced by the corresponding arm powers $p_{x y}$. To ensure stable operation, the pulsating arm energies are averaged. As shown in section II-E the arm energies pulsate with different frequencies. To simplify the controller design the energies are filtered and the average value is controlled to zero [8]. There are several ways of balancing the arm energies within the system that have to be applied depending on the operating point [5].

\section{Simplified State-of-the-Art Control Scheme}

In [8] a detailed description of the M3C control strategy is given. A simplified structure can be seen in fig. 3. The control approach presented in this paper can be used in addition to this scheme. As described in sections II-B and II-C the aim is to control the decoupled input, output and internal currents. At the same time all arm energies have to be balanced.

The modulator sums up all measured cell voltages to 9 arm voltages $v_{\mathrm{C}, \mathrm{xy}}$. The arm energies are calculated by eq. (2) and all components are transformed according to eq. (1) to decouple the system. The control is a cascaded scheme with an inner current controller and an overlaying energy controller for each of the decoupled components. The transformed arm energies pulsate with different frequencies (see section II-E). The 9 energy controllers calculate the necessary power to balance the energy. The overall energy $w_{00}$ of the system then is controlled with the input currents $i_{\text {in } \alpha \beta}$. The 8 remaining components can be balanced by using a combination of one of the four internal currents $i_{\mathrm{i} 12 \alpha \beta}$ and either the input or the output voltage. In addition internal currents can be injected to compensate occurring energy pulsation within the system as described in section III. The output side of the converter 


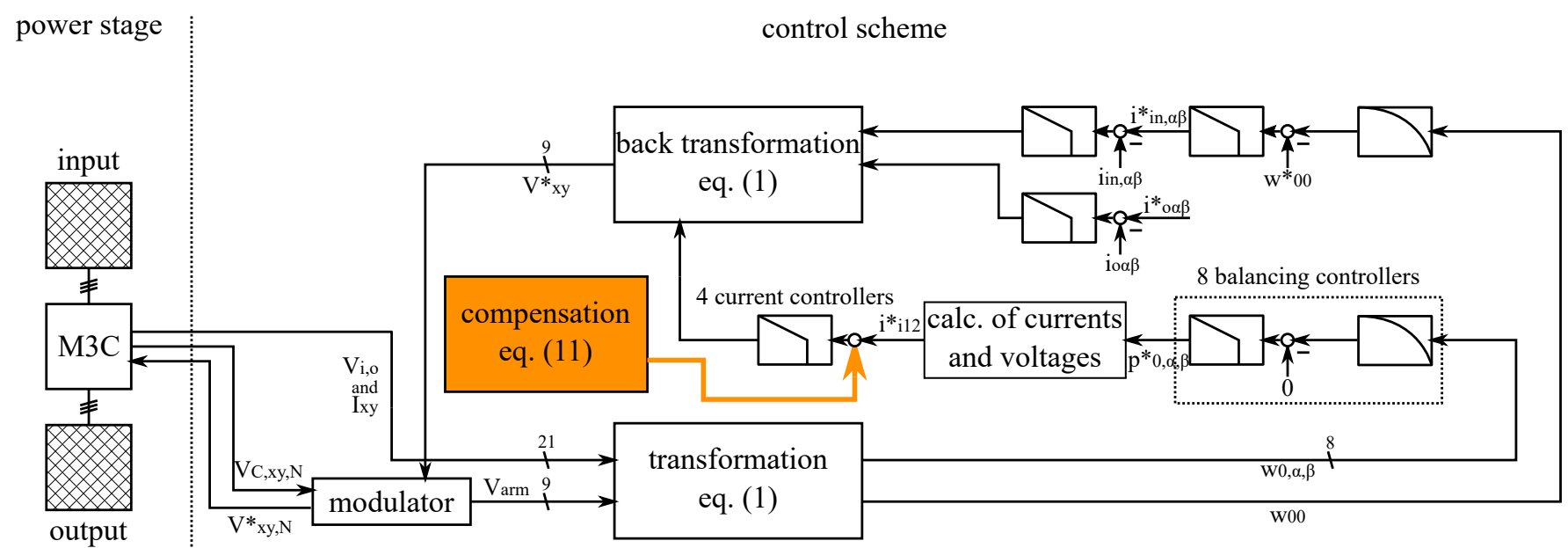

Fig. 3. State-of-the-Art cascaded control scheme of the Modular Multilevel Matrix Converter [8] with additional compensation of energy pulsations caused by the input side

can be controlled independently. The desired values $v_{\mathrm{xy}}$ for the arm voltages are transformed to calculate the modulation index for each arm. The modulator triggers the semiconductors in each arm. This control scheme allows a simple decoupled controller design. [8] shows that this control approach is valid for the whole frequency range.

With the new approach presented in section III improvements can be made to reduce the energy pulsation when the output frequency is higher than the input frequency. The power components of the system have to be analyzed carefully. Set points for additional internal currents are determined to reduce the energy ripple (see fig. 3, orange block).

\section{E. Power Components of the System}

The internal currents of the $\mathrm{M} 3 \mathrm{C}$ can be set to 0 during steady state operation with different input and output frequencies ([1], [8]). They can also be controlled in a way to compensate occurring energy pulsations (see section III-A). To control the currents, the corresponding arm voltages $v_{x y}$ are used. In order to determine the energy pulsation the arm power has to be investigated. It is assumed that the energy in one arm is distributed equally among all cell capacitors due to the internal modulation scheme. Therefore only the distribution among the arms has to be considered. The arm power can be calculated by $p_{x y}=v_{x y} \cdot i_{x y}$. Assuming sinusoidal currents and voltages with an amplitude $A$ and a phase angle $\gamma$, the 9 arm powers are transformed by $T_{\mathrm{p}}$. Neglecting losses, the 9 resulting power components represent the power exchanged among the converter arms and the overall power $p_{00}$. Interpreting the $\alpha$ - and $\beta$-components as $\mathbb{R}$ and $\mathbb{I}$ part of a complex space vector the decoupled power components are

$$
\begin{aligned}
\underline{p}_{\alpha \beta 0}= & \frac{1}{6} \hat{v}_{\text {in }} \hat{\imath}_{\text {in }} e^{-\mathrm{j}\left(2 \gamma_{\text {in }}+\varphi_{\text {in }}\right)}-\frac{1}{2} \hat{v}_{\text {out }} \hat{\imath}_{\mathrm{i} 1} e^{\mathrm{j}\left(\gamma_{\text {out }}-\gamma_{\mathrm{i} 1}\right)} \\
& -\frac{1}{2} \hat{v}_{\text {out }} \hat{\imath}_{\mathrm{i} 2} e^{\mathrm{j}\left(-\gamma_{\text {out }}+\gamma_{\mathrm{i} 2}\right)}-\frac{1}{6} \hat{v}_{0} \hat{\imath}_{\mathrm{in}} e^{\mathrm{j}\left(\gamma_{\text {in }}+\varphi_{\text {in }} \pm \gamma_{0}\right)} \\
\underline{p}_{0 \alpha \beta}= & \frac{1}{6} \hat{v}_{\text {out }} \hat{\imath}_{\text {out }} e^{-\mathrm{j}\left(2 \gamma_{\text {out }}+\varphi_{\text {out }}\right)}+\frac{1}{2} \hat{v}_{\text {in }} \hat{\imath}_{\mathrm{i} 1} e^{\mathrm{j}\left(\gamma_{\text {in }}+\gamma_{\mathrm{i} 1}\right)}
\end{aligned}
$$

$$
\begin{aligned}
& +\frac{1}{2} \hat{v}_{\text {in }} \hat{\imath}_{\mathrm{i} 2} e^{\mathrm{j}\left(-\gamma_{\mathrm{in}}+\gamma_{\mathrm{i} 2}\right)}-\frac{1}{6} \hat{v}_{0} \hat{\imath}_{\text {out }} e^{\mathrm{j}\left(\gamma_{\text {out }}+\varphi_{\text {out }} \pm \gamma_{0}\right)} \\
& \underline{p}_{\text {i1 }}=\frac{1}{6} \hat{v}_{\text {in }} \hat{\imath}_{\text {out }} e^{\mathrm{j}\left(\gamma_{\text {out }}+\varphi_{\text {out }}-\gamma_{\text {in }}\right)}-\frac{1}{6} \hat{v}_{\text {out }} \hat{\imath}_{\text {in }} e^{\mathrm{j}\left(\gamma_{\text {out }}-\left(\gamma_{\text {in }}+\varphi_{\text {in }}\right)\right)} \\
& +\frac{1}{2} \hat{v}_{\mathrm{in}} \hat{\imath}_{\mathrm{i} 2} e^{\mathrm{j}\left(-\gamma_{\mathrm{in}}+\gamma_{\mathrm{i} 2}\right)}-\frac{1}{2} \hat{v}_{\mathrm{out}} \hat{\imath}_{\mathrm{i} 2} e^{\mathrm{j}\left(-\gamma_{\mathrm{out}}+\gamma_{\mathrm{i} 2}\right)} \\
& -\frac{1}{2} \hat{v}_{0} \hat{\imath}_{\mathrm{i} 1} e^{\mathrm{j}\left(\gamma_{0} \pm \gamma_{\mathrm{i} 1}\right)} \\
& \underline{p}_{\mathrm{i} 2}=\frac{1}{6} \hat{v}_{\text {in }} \hat{\imath}_{\text {out }} e^{\mathrm{j}\left(\gamma_{\text {out }}+\varphi_{\text {out }}+\gamma_{\text {in }}\right)}-\frac{1}{6} \hat{v}_{\text {out }} \hat{l}_{\text {in }} e^{\mathrm{j}\left(\gamma_{\text {out }}+\gamma_{\text {in }}+\varphi_{\text {in }}\right)} \\
& +\frac{1}{2} \hat{v}_{\mathrm{in}} \hat{\imath}_{\mathrm{i} 1} e^{\mathrm{j}\left(-\gamma_{\mathrm{in}}+\gamma_{\mathrm{i} 1}\right)}-\frac{1}{2} \hat{v}_{\mathrm{out}} \hat{\mathrm{i}}_{\mathrm{i} 1} e^{\mathrm{j}\left(-\gamma_{\mathrm{out}}-\gamma_{\mathrm{i} 1}\right)} \\
& -\frac{1}{2} \hat{v}_{0} \hat{\imath}_{\mathrm{i} 2} e^{\mathrm{j}\left(\gamma_{0} \pm \gamma_{\mathrm{i} 2}\right)} \\
& \underline{p}_{00}=\frac{1}{6} \hat{v}_{\text {in }} \hat{\imath}_{\text {in }} \cos \left(\varphi_{\text {in }}\right)-\frac{1}{6} \hat{v}_{\text {out }} \hat{\imath}_{\text {out }} \cos \left(\varphi_{\text {out }}\right)
\end{aligned}
$$

The energy in each arm is given by

$$
w_{x y}=W_{0}+\underbrace{\int_{t_{0}}^{t_{0}+T} p_{x y} \mathrm{~d} t}_{\stackrel{!}{=} 0} \stackrel{!}{=} \text { const. }
$$

During steady state operation of the M3C each power component has to be 0 in average to ensure stable operation as shown in eq. (8).

\section{COMpensation of Power COMPonents}

\section{A. Pulsating Power when the Output Frequency is Greater than the Input Frequency}

When driving a high frequency load or grid, eqs. (3) to (7) have to be analyzed to find degrees of freedom to reduce the energy pulsations. The analysis is done for positive output frequencies but can also be applied to negative output frequencies. The zero sequence voltage $v_{0}$ and the second internal current $\underline{i}_{\mathrm{i} 2}$ are set to 0 because they are not needed for balancing the energy when the output frequency is greater 
than the input frequency. Losses are neglected. The resulting terms of interests are

$$
\begin{aligned}
\underline{p}_{\alpha \beta 0}= & \frac{1}{6} \hat{v}_{\text {in }} \hat{\imath}_{\text {in }} e^{-\mathrm{j}\left(2 \gamma_{\text {in }}+\varphi_{\text {in }}\right)}-\frac{1}{2} \hat{v}_{\text {out }} \hat{\imath}_{\mathrm{i} 1} e^{\mathrm{j}\left(\gamma_{\text {out }}-\gamma_{\mathrm{i} 1}\right)} \\
\underline{p}_{\mathrm{i} 2}= & \frac{1}{6} \hat{v}_{\text {in }} \hat{\imath}_{\text {out }} e^{\mathrm{j}\left(\gamma_{\text {out }}+\varphi_{\text {out }}+\gamma_{\text {in }}\right)}-\frac{1}{6} \hat{v}_{\text {out }} \hat{\imath}_{\text {in }} e^{\mathrm{j}\left(\gamma_{\text {out }}+\gamma_{\text {in }}+\varphi_{\text {in }}\right)} \\
& +\frac{1}{2} \hat{v}_{\text {in }} \hat{\imath}_{\text {i1 } 1} e^{\mathrm{j}\left(-\gamma_{\text {in }}+\gamma_{\text {i1 }}\right)}-\frac{1}{2} \hat{v}_{\text {out }} \hat{\imath}_{\mathrm{i} 1} e^{\mathrm{j}\left(-\gamma_{\text {out }}+\gamma_{\mathrm{i} 2}\right)}
\end{aligned}
$$

Equation (9) contains a power term that oscillates with twice the input frequency (left term in eq. (9)). This term causes the main energy pulsations in the converter arms when the output frequency is greater than the input frequency. It can be compensated with the second part of the term in eq. (9). Therefore an additional current $\hat{\imath}_{\mathrm{i} 1}$ is induced. The resulting component to compensate the pulsations is

$$
\underline{p}_{\alpha \beta 0, \text { comp }}=-\frac{1}{2} \hat{v}_{\text {out }} \hat{\imath}_{\mathrm{i} 1} e^{\mathrm{j}\left(\gamma_{\text {out }}-\gamma_{\mathrm{i} 1}\right)}
$$

Equation (11) must meet the requirements from eqs. (12) and (13) to compensate the pulsating component.

$$
\begin{aligned}
\hat{\imath}_{\mathrm{i} 1} & =k_{2 \mathrm{~h}} \frac{1}{3} \hat{\imath}_{\mathrm{in}} \\
\gamma_{\mathrm{i} 1} & =\gamma_{\text {out }}+2 \gamma_{\mathrm{in}}+\varphi_{\mathrm{in}}
\end{aligned}
$$

The current $\hat{\imath}_{\mathrm{i} 1}$ can cause additional pulsations in eq. (10) that have to be taken into account when choosing the degree of compensation. $k_{2 \mathrm{~h}}$ describes this degree of compensation. With the compensation ratio $k_{2 \mathrm{~h}}=1$ the power component $\underline{p}_{\alpha \beta 0}$ is 0 . The same applies to negative output frequencies. To reduce the pulsation in power term $\underline{p}_{\alpha \beta 0}$ it is more suitable to use internal current $\underline{i}_{\mathrm{i} 2}$ and set $\underline{i}_{\mathrm{i} 1}$ to 0 [5]. The amplitude and phase requirements for the compensating current $\underline{i}_{\text {i2 }}$ are

$$
\begin{aligned}
\hat{\imath}_{\mathrm{i} 2} & =k_{2 \mathrm{~h}, \text { neg }} \frac{1}{3} \hat{\imath}_{\text {in }} \\
\gamma_{\mathrm{i} 2} & =\gamma_{\text {out }}+2 \gamma_{\text {in }}+\varphi_{\text {in }}
\end{aligned}
$$

Pulsating terms with twice the input frequency can be reduced for negative output frequencies with the internal current $\underline{i}_{\mathrm{i} 2}$. A complete compensation of the terms with a frequency $\omega=$ $2 \omega_{\text {in }}$ is not necessarily optimal regarding the overall energy pulsation as shown in section III-B. The optimal value for the compensation factor $k_{2 \mathrm{~h}}$ has to be analyzed for each operating point. For different points of operation different degrees of compensation can lead to an overall reduced energy ripple. With the compensation of the pulsating power components, the converter's pulsating energies and therefore the voltage ripple in each cell capacitor can be reduced leading to a smaller cell capacitor for the $\mathrm{M} 3 \mathrm{C}$ cell.

\section{B. Compensation of Components Pulsating with Twice the Input Frequency}

To simplify the analysis the power components are normalized to the converter's nominal power $S_{\mathrm{N}}$. The voltage ratio $\frac{\hat{v}_{\text {in }}}{\hat{v}_{\text {out }}}$ between input and output side is set to 1 . Losses are neglected and therefore $\hat{\imath}_{\text {out }}=\hat{\imath}_{\text {in }} \cdot \frac{\cos \left(\varphi_{\text {in }}\right)}{\cos \left(\varphi_{\text {out }}\right)}$. The angle $\gamma$ equals $\omega t$ and the ratio between input frequency and output frequency is $\nu=\frac{\omega_{\text {out }}}{\omega_{\text {in }}}$. As an example the resulting energy for arm 11 at the same power factor on input and output side (here: $\varphi_{\mathrm{i}}=\varphi_{\mathrm{o}}=1$ ) is calculated with eq. (8)

$$
\begin{aligned}
w_{11}= & \int p_{11} \mathrm{~d} t \\
= & w_{\alpha 0}+w_{0 \alpha}+w_{\mathrm{i} 1 \alpha}+w_{\mathrm{i} 2 \alpha}+W_{0} \\
= & \frac{1}{9}\left[\frac{\sin \left(2 \omega_{\text {in }} t\right)}{2 \omega_{\text {in }}} \cdot\left(1-k_{2 \mathrm{~h}}\right)\right. \\
& +\frac{\sin \left((\nu+3) \omega_{\text {in }} t\right)}{(\nu+3) \omega_{\text {in }}} \cdot k_{2 \mathrm{~h}}-\frac{\sin \left(2 \nu \omega_{\text {in }} t\right)}{2 \nu \omega_{\text {in }}} \\
& \left.+k_{2 \mathrm{~h}} \cdot\left(\frac{2 \sin \left((\nu+1) \omega_{\text {in }} t\right)-\sin \left(2(\nu+1) \omega_{\text {in }} t\right)}{2(\nu+1) \omega_{\text {in }}}\right)\right] \\
& +W_{0}
\end{aligned}
$$

All arm powers can be calculated in the same way using eq. (8). As a result the energy pulsation in each arm is given. The total energy pulsation of one arm is calculated for different compensation factors $k_{2 \mathrm{~h}}$. Different power factors can be considered as well but the effect of reduction is largest at unity power factor on input and output side.

\section{Analytical Results of Reducing the Total Energy Pulsation}

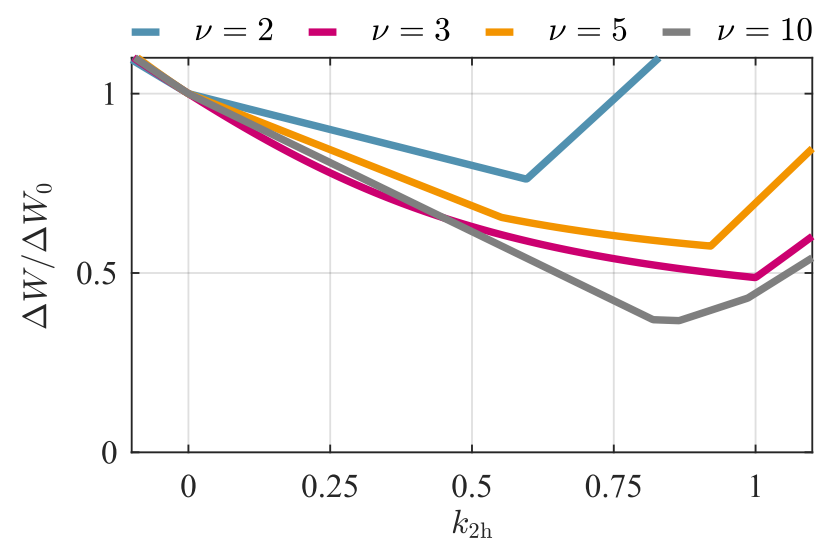

Fig. 4. Reduction of energy pulsation for a varying compensation factor $k_{2 \mathrm{~h}}$ and different frequency ratios $\nu$

Figure 4 shows numerical results of the energy pulsation in the arms of the converter (eq. (16)). The energy pulsation is shown for different frequency ratios $\nu$. Depending on the reduction factor $k_{2 \mathrm{~h}}$ the total pulsation $\Delta W=\max \left(W_{x y}\right)-$ $\min \left(W_{x y}\right)$ can be reduced. The terms are compared to the operation without any compensation (compensation factor $k_{2 \mathrm{~h}}=0$ ). The maximum of reduction is not necessarily achieved for a factor of $k_{2 \mathrm{~h}}=1$ due to the complex superposition of sinusoidal terms. As can be seen in fig. 4, values for the degree of compensation $k_{2 \mathrm{~h}} \leq 0$ or $k_{2 \mathrm{~h}} \geq 1$ are not feasible at any operation point. The results show that the total energy pulsation can be reduced significantly by inducing additional currents. Therefore the total capacity and volume can be reduced. These currents however cause additional losses that have to be investigated further. 
TABLE I

SETUP PARAMETERS OF A M3C FOR CALCULATING ADDITIONAL SEMICONDUCTOR LOSSES

\begin{tabular}{cc}
\hline \hline nominal power & $10 \mathrm{MVA}$ \\
nominal voltage & $4.16 \mathrm{kV}$ \\
cells per arm & 8 \\
capacitance per cell & $6000 \mu \mathrm{F}$ \\
nominal voltage per cell & $1000 \mathrm{~V}$ \\
input frequency & $50 \mathrm{~Hz}$ \\
power factor & $\cos \left(\varphi_{\text {in }}\right)=\cos \left(\varphi_{\text {out }}\right)=1$ \\
control frequency & $20 \mathrm{kHz}$ \\
power modules & Infineon $\mathrm{FF} 1400 \mathrm{R} 17 \mathrm{IP} 4[9]$ \\
cell capacitor energy density & $270 \mathrm{~J} / 1$ \\
\hline \hline
\end{tabular}

\section{Additional Losses}

An example configuration of a Modular Multilevel Matrix Converter is used to identify additional losses caused by the compensating currents in the system. A theoretical setup is used, since there is no M3C system commercially available yet. Reducing the energy ripple allows a reduction of the cell capacitors and therefore increases the energy density. The costs of the M3C system can be reduced. Table I shows the parameters of the system. The conduction and switching losses are computed with a simplified converter model based on [10]. The losses are calculated for one cell of the M3C system. It is assumed that the energy and losses are distributed equally among all converter arms. The pulsation for one arm can be calculated with eq. (16). The internal modulation of one arm is considered to stress each cell evenly. During one control period the cell capacitor voltage is assumed to be constant. The reduction of energy pulsation in each cell allows

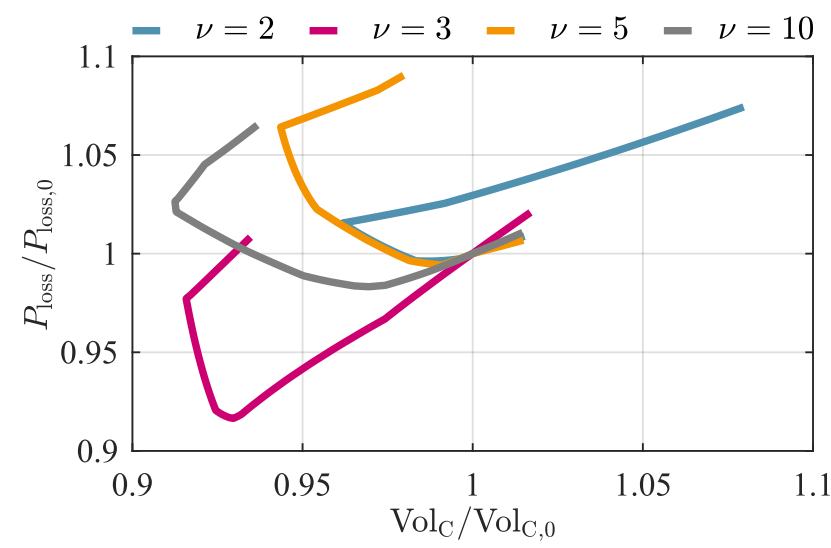

Fig. 5. Semiconductor losses compared to the volume change of each cell for varying reduction factor $k_{2 \mathrm{~h}}$ and different frequency ratios $\nu$

a reduction of the cell capacitance and the volume of the cells. Depending on the degree of reduction $k_{2 \mathrm{~h}}$ the design can be adapted according to fig. 5. The plot depicts the semiconductor losses in comparison to the volume of each cell for varying compensation factor $k_{2 \mathrm{~h}}$ with $-0.1 \leq k_{2 \mathrm{~h}} \leq 1.1$. A minimum of the total volume of the cell can be achieved by applying the factor $k_{2 \mathrm{~h}}$ of minimal energy pulsation from fig. 4. The losses are not linearly connected to the degree of compensation $k_{2 \mathrm{~h}}$ because of the superposition of the cell capacitor voltage pulsations.

\section{Simulation Results}

The analysis is verified by simulation for a laboratory scaled prototype of the $\mathrm{M} 3 \mathrm{C}$. The parameters of the system are given in table II.

TABLE II

PARAMETERS OF THE LABORATORY SCALED PROTOTYPE OF THE M3C

\begin{tabular}{cc}
\hline \hline input voltage & $230 \mathrm{~V}$ \\
input current & $56 \mathrm{~A}$ \\
output voltage & $345 \mathrm{~V}$ \\
cells per arm & 5 \\
capacitance per cell & $1100 \mu \mathrm{F}$ \\
nominal voltage per arm & $930 \mathrm{~V}$ \\
input frequency & $50 \mathrm{~Hz}$ \\
output frequency & $500 \mathrm{~Hz}$ \\
control frequency & $8 \mathrm{kHz}$ \\
\hline \hline
\end{tabular}

\section{A. Steady State Operation}

The pulsating energies caused by the input side are compensated by injecting additional internal currents. The injection leads to a reduction of the overall voltage ripple in the cell capacitors. The results are shown in fig. 6. At $t=0.02 \mathrm{~s}$ the total compensation of the energy pulsations is activated. Figure 6 a) shows the arm voltages. The setpoint for the average of $v_{\mathrm{C}, 00} \approx 930 \mathrm{~V}$ is held over the whole time range. The pulsation is reduced by additional currents. Figure $6 \mathrm{~b}$ ), f) and h) show the grid voltages and currents on the input and the output sides. The frequency ratio is $\nu=\frac{f_{\text {out }}}{f_{\text {in }}}=$ $\frac{500 \mathrm{~Hz}}{50 \mathrm{~Hz}}=10$. The capacitor voltages are transformed and converted to energies. Figure $6 \mathrm{c}$ ), e), g) and i) show the decoupled transformed converter arm energies. Figure $6 \mathrm{c}$ ) depicts the energy pulsations caused by the input side. The additional injected currents shown in Figure $6 \mathrm{j}$ ) yield to a total compensation of the pulsations. Figure $6 \mathrm{~d}$ ) show the arm currents for each of the 9 converter arms. It can be seen that the compensation works perfectly. The energy pulsation in $w_{\alpha \beta 0}$ is 0 after the internal currents are injected. There are additional pulsations in components $w_{0 \alpha \beta}$ as well as $w_{\mathrm{i} 1 \alpha \beta}$ and the arm currents are increased. The overall voltage pulsation however can be reduced by $68 \%$. Therefore, the arm RMScurrents are increased by $14 \%$. The compensation introduced in this paper is verified by simulation results.

\section{B. Different Power Factors}

The power factor on the input side can be varied resulting in a lower degree of possible energy pulsation reduction. Figure 7 depicts the possible reduction for different phase shifts $\varphi_{\text {in }}$ on the input side. For a power factor of $\cos \left(\varphi_{\text {in }}\right)= \pm 1$ the remaining pulsation is only about $31 \%$ of the pulsation without any compensation. For a phase shift of $\varphi_{\text {in }}= \pm 90^{\circ}$ 

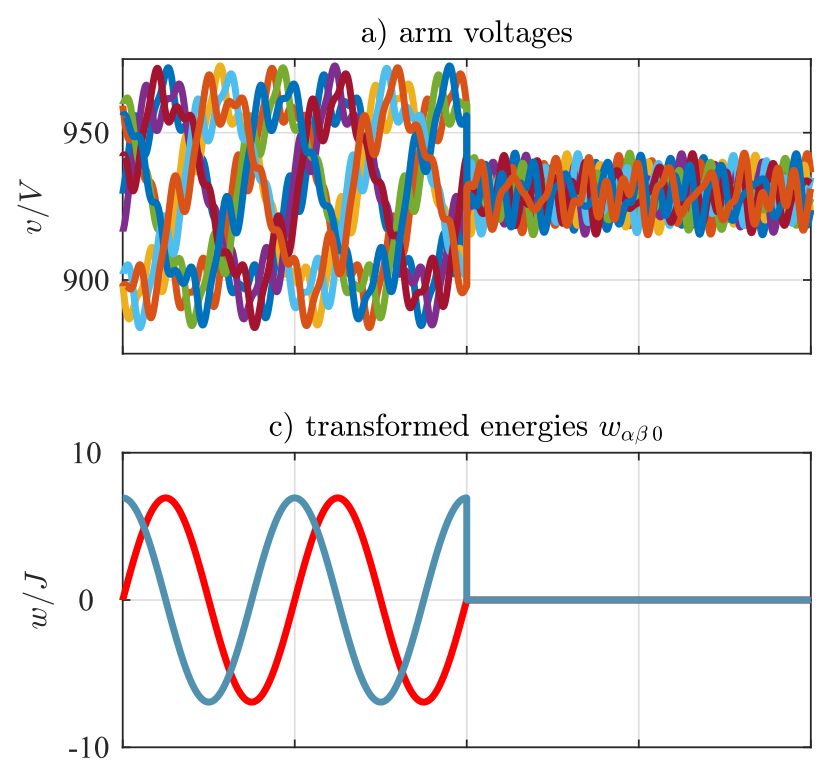

e) transformed energies $w_{0 \alpha \beta}$

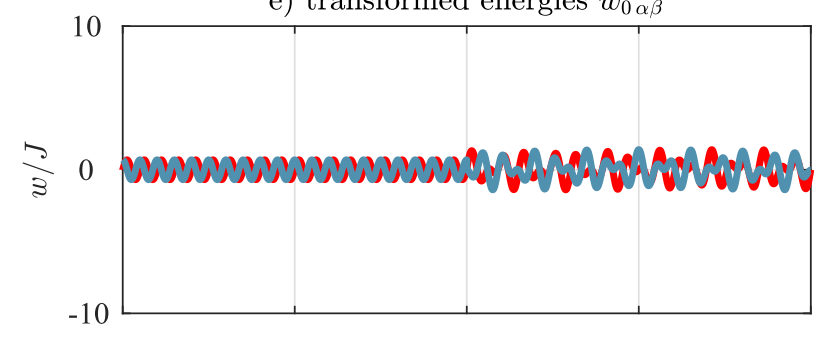

g) transformed energies $w_{\mathrm{i} 1 \alpha \beta}$

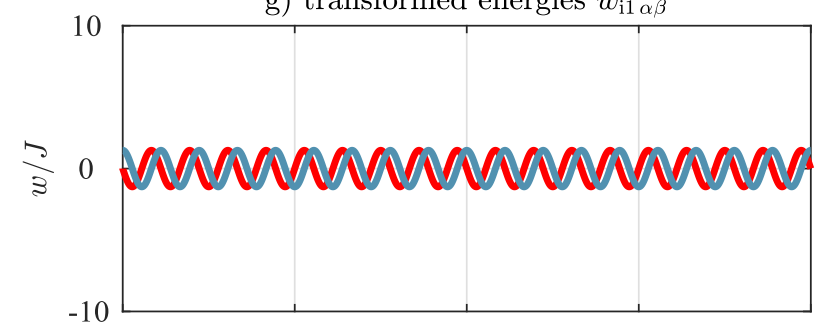

i) transformed energies $w_{\mathrm{i} 2 \alpha \beta}$

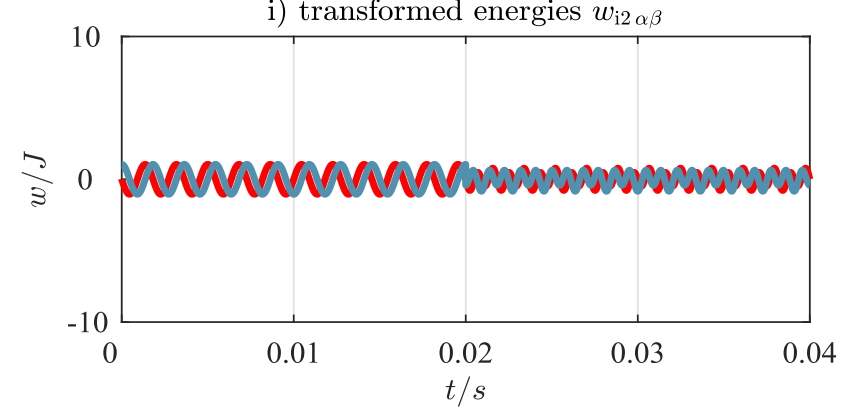

b) grid voltages $v_{\text {in } \alpha \beta}, v_{\text {out } \alpha \beta}$
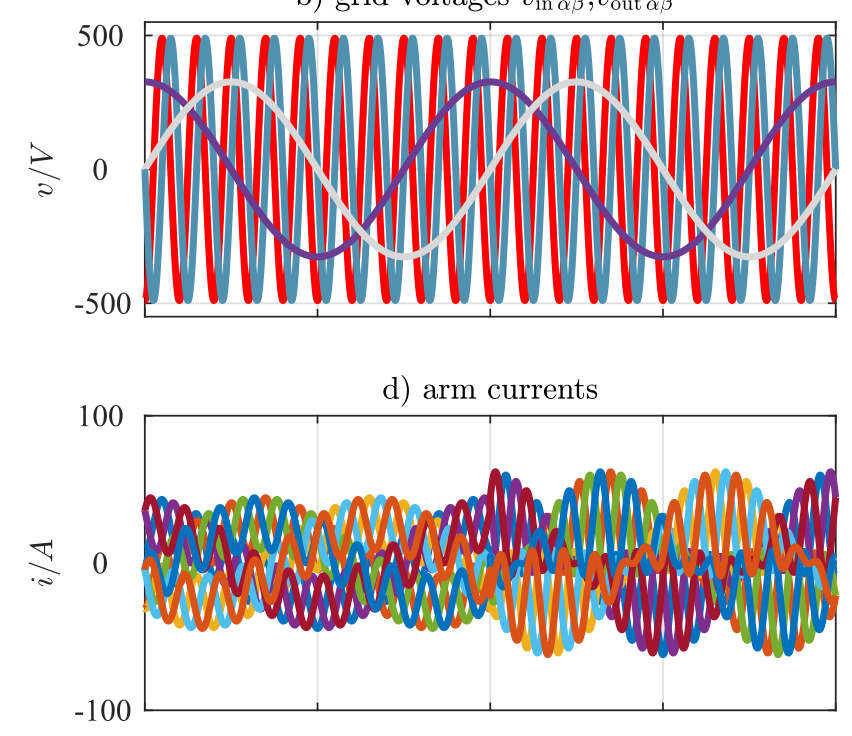

f) input currents $i_{\operatorname{in} \alpha \beta}$

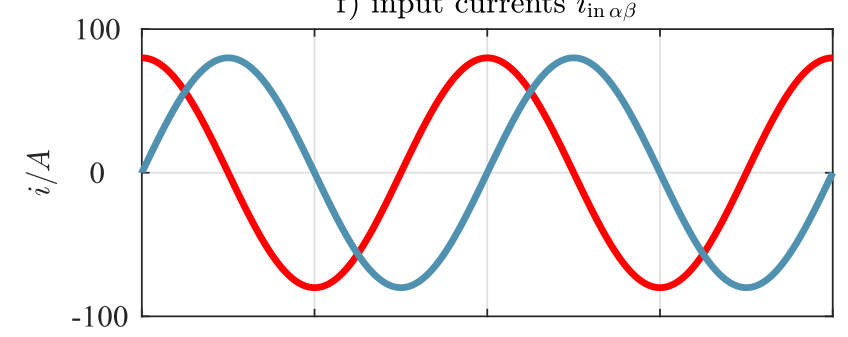

h) output currents $i_{\text {out } \alpha \beta}$
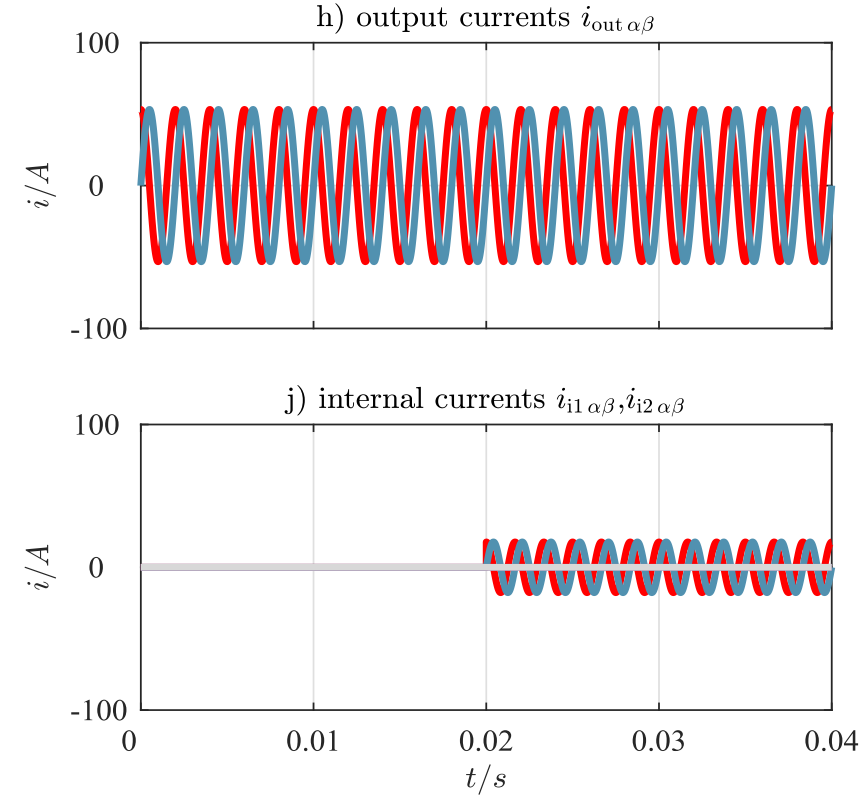

Fig. 6. Simulation results of energy pulsation compensation for high output frequencies. a) capacitor voltages per arm, b) input and output voltages, c) transformed energies $w_{\alpha \beta 0}$ with total compensation, d) arm currents, e) transformed energies $w_{0 \alpha \beta}$, f) input currents, g) transformed energies $w_{\mathrm{i} 1 \alpha \beta}$, h) output currents, i) transformed energies $w_{\mathrm{i} 2 \alpha \beta}$, j) internal currents with compensation currents

the resulting pulsation is only reduced down to $45 \%$ of the original pulsation. Simulations for negative output frequencies and for a varying phase shift on the output side were also done but not presented due to space requirements. Nevertheless the compensation works for all operation points of the M3C. The results will be verified with the laboratory setup in future work. 
$\Delta w / \Delta w_{0}$ for varying $\varphi$

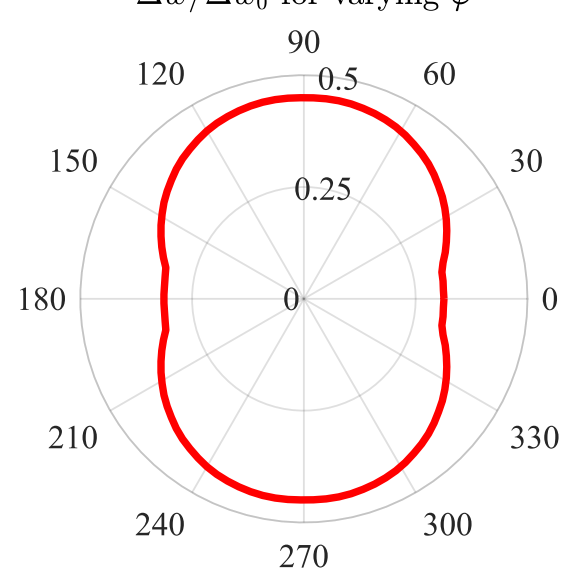

Fig. 7. Reduction of energy pulsation for a varying phase shift $\varphi_{\text {in }}$ on the input side

\section{CONCLUSION}

This paper describes a control strategy for Modular Multilevel Matrix Converters (M3C) with high output frequencies to reduce the energy pulsation in each arm. The major energy pulsation at operating points with an output frequency larger than the input frequency $\omega_{\text {out }}>>\omega_{\text {in }}$ occurs with twice the input frequency $2 \omega_{\text {in }}$ and can be compensated with additional internal currents that are neither effective on the input side nor the output side. To determine the necessary currents, the measured current and voltage values of the converter are transformed to get a decoupled system. With this analysis, the currents and resulting reduction of the energy pulsations can be calculated. Depending on the ratio $\nu$ between the input frequency and the output frequency the degree of compensation $k_{2 \mathrm{~h}}$ can be determined to get a minimal energy pulsation. This compensation can be performed for positive and negative output frequencies. The additional compensating currents cause additional semiconductor losses. With a theoretical setup of a medium voltage M3C the additional losses are calculated with a simplified switching model of the converter. The results show a reduction of the cell volume and therefore costs over additional losses. The M3C cells can be designed according to this results. The theoretical analysis of the energy pulsation compensation are finally verified by simulations for a laboratory scaled prototype to illustrate the effect of the reduction.

$$
\begin{aligned}
T_{\mathrm{i}}= & T_{\mathrm{v}}=T_{\mathrm{p}} \\
= & {\left[\begin{array}{ccccc}
\frac{2}{9} & -\frac{1}{9} & -\frac{1}{9} & -\frac{1}{9} & \frac{2}{9} \\
0 & -\frac{\sqrt{3}}{9} & \frac{\sqrt{3}}{9} & \frac{\sqrt{3}}{9} & 0 \\
\frac{2}{3} & -\frac{2}{3} & -\frac{1}{3} & \frac{2}{3} & -\frac{2}{3} \\
\frac{2}{9} & -\frac{1}{9} & -\frac{1}{9} & -\frac{1}{9} & -\frac{1}{9} \\
0 & \frac{\sqrt{3}}{9} & -\frac{\sqrt{3}}{9} & \frac{\sqrt{3}}{9} & -\frac{\sqrt{3}}{9} \\
0 & \frac{\sqrt{3}}{3} & -\frac{\sqrt{3}}{3} & 0 & \frac{\sqrt{3}}{3} \\
\frac{2}{3} & \frac{2}{3} & \frac{2}{3} & -\frac{1}{3} & -\frac{1}{3} \\
0 & 0 & 0 & \frac{\sqrt{3}}{3} & \frac{\sqrt{3}}{3} \\
\frac{1}{9} & \frac{1}{9} & \frac{1}{9} & \frac{1}{9} & \frac{1}{9} \\
& -\frac{1}{9} & -\frac{1}{9} & -\frac{1}{9} & \frac{2}{9} \\
& -\frac{\sqrt{3}}{9} & -\frac{\sqrt{3}}{9} & \frac{\sqrt{3}}{9} & 0 \\
& -\frac{1}{3} & \frac{2}{3} & -\frac{2}{3} & -\frac{1}{3} \\
& \frac{2}{9} & -\frac{1}{9} & \frac{2}{9} & -\frac{1}{9} \\
0 & -\frac{\sqrt{3}}{9} & 0 & \frac{\sqrt{3}}{9} \\
& -\frac{\sqrt{3}}{3} & 0 & \frac{\sqrt{3}}{3} & -\frac{\sqrt{3}}{3} \\
& -\frac{1}{3} & -\frac{1}{3} & -\frac{1}{3} & -\frac{1}{3} \\
& \frac{\sqrt{3}}{3} & -\frac{\sqrt{3}}{3} & -\frac{\sqrt{3}}{3} & -\frac{\sqrt{3}}{3} \\
\frac{1}{9} & \frac{1}{9} & \frac{1}{9} & \frac{1}{9}
\end{array}\right] }
\end{aligned}
$$

[1] W. Kawamura and H. Akagi, "Control of the modular multilevel cascade converter based on triple-star bridge-cells (mmcc-tsbc) for motor drives," in 2012 IEEE Energy Conversion Congress and Exposition (ECCE), Sept 2012, pp. 3506-3513.

[2] F. Kammerer, D. Braeckle, M. Gommeringer, M. Schnarrenberger, and M. Braun, "Operating performance of the modular multilevel matrix converter in drive applications," in Proceedings of PCIM Europe 2015, International Exhibition and Conference for Power Electronics, Intelligent Motion, Renewable Energy and Energy Management, May 2015, pp. $1-8$.

[3] A. J. Korn, M. Winkelnkemper, P. Steimer, and J. W. Kolar, "Direct modular multi-level converter for gearless low-speed drives," in Proceedings of the 2011 14th European Conference on Power Electronics and Applications, Aug 2011, pp. 1-7.

[4] D. Braeckle, F. Kammerer, M. Schnarrenberger, M. Hiller, and M. Braun, "A modular multilevel matrix converter for high speed drive applications," in PCIM Europe 2016; International Exhibition and Conference for Power Electronics, Intelligent Motion, Renewable Energy and Energy Management, May 2016, pp. 1-8.

[5] F. Kammerer, M. Gommeringer, J. Kolb, and M. Braun, "Energy balancing of the modular multilevel matrix converter based on a new transformed arm power analysis," in 2014 16th European Conference on Power Electronics and Applications, Aug 2014, pp. 1-10.

[6] W. Kawamura, M. Hagiwara, and H. Akagi, "A broad range of frequency control for the modular multilevel cascade converter based on triple-star bridge-cells (mmcc-tsbc)," in 2013 IEEE Energy Conversion Congress and Exposition, Sept 2013, pp. 4014-4021.

[7] K. Ilves, L. Bessegato, and S. Norrga, "Comparison of cascaded multilevel converter topologies for ac/ac conversion," in 2014 International Power Electronics Conference (IPEC-Hiroshima 2014 - ECCE ASIA), May 2014, pp. 1087-1094.

[8] F. Kammerer, "Systemanalyse und regelung des modularen multilevel matrix umrichters als antriebsumrichter," dissertation, Karlsruhe Institute of Technology, 2016.

[9] PrimePACK3 module with trench/fieldstop IGBT4 and Emitter Controlled Diode and NTC, Infineon Technologies AG, 11 2013, rev. 2.4.

[10] M. Schnarrenberger, F. Kammerer, D. Brckle, and M. Braun, "Cell design of a square-wave powered 1ac-3ac modular multilevel converter low voltage prototype," in 2016 18th European Conference on Power Electronics and Applications (EPE'16 ECCE Europe), Sept 2016, pp. $1-11$. 